\title{
Relações entre Elementos da Gestão Pública e a Corrupção nos Estados Brasileiros
}

\section{Relationships between Elements of Public Management and Corruption in the Brazilian States}

\author{
Anderson de Oliveira Reis ${ }^{1}$ \\ Fernanda Maria de Almeida ${ }^{2}$
}

\section{Resumo}

A corrupção tem sido intensamente debatida nas discussões sobre a administração pública por causar corrosão nas receitas públicas e ineficiência na gestão, principalmente na área social. Devido às consequências negativas apresentadas por esse fenômeno, propõem-se, com este estudo, medir o nível de corrupção nos estados brasileiros por meio da construção de um índice estadual de corrupção (ICE) e verificar possíveis relações entre investimentos públicos, saúde, educação, endividamento e a corrupção. A análise compreendeu o período de 2010 a 2012, e utilizou-se das metodologias de análise fatorial e teste de média de Kruskal-Wallis. Os resultados apontam que os estados do Nordeste e do Sudeste apresentam, em média, maiores níveis de corrupção. Já os estados do Sul e os estados do Acre e Rondônia são possíveis casos exemplares em práticas de controle da corrupção, pois apresentam menor incidência do fenômeno.

Palavras-chave: corrupção; gastos públicos; administração pública.

\begin{abstract}
Corruption has been intensely debated in discussions about public administration because it causes corrosion in public revenues and inefficiency in management, especially in the social area. Due to the negative consequences presented by this phenomenon, it is proposed, with this study, to measure the level of corruption in Brazilian states through the construction of a state corruption index (ICE) and to verify possible relations between public investments, health, education, indebtedness, and corruption. The analysis covered the period from 2010 to 2012 and used the factor analysis methodologies and the Kruskal-Wallis mean test. The results show that the states of the Northeast and Southeast have, on average, higher levels of corruption. The southern states and the states of Acre and Rondônia are exemplary cases in corruption control practices, as they have a lower incidence of the phenomenon.
\end{abstract}

Keywords: corruption; public spending; public administration. Doutorando em Administração pela Universidade Federal de Viçosa (UFV). 


\section{INTRODUÇÃO}

A demanda crescente da sociedade por serviços públicos e a escassez de recursos exigem dos governos eficiência na gestão (Zani \& Spinelli, 2010). A necessidade de manter o equilíbrio fiscal e de oferecer serviços públicos de qualidade tornou a eficiência um dos pontos centrais de discussão na administração pública (Pascarelli Filho, 2011). A corrupção é vista como um elemento perversivo do ponto de vista socioeconômico, uma vez que causa deterioração dos recursos públicos e pode comprometer o oferecimento dos serviços à população e a eficiência na gestão pública (Lopes \& Toyoshima, 2013; Filgueiras, 2009). Nas, Price \& Weber (1986) definem corrupção como qualquer forma de ação ilícita do poder público ou de autoridade para benefício próprio.

Melo (2010) considera que atender às demandas sociais com recursos escassos faz parte do cotidiano dos gestores públicos e que, quando se discute desempenho na ação estatal, a corrupção é tema recorrente devido a seus efeitos negativos. Ao beneficiar os inescrupulosos e aqueles que possuem maior nível de network, ou seja, aqueles que possuem maior rede de contatos sociais, tanto no âmbito público como no privado, o que lhes permite ter mais informações, e utilizam dessa rede e informações para lesionar o patrimônio público, em detrimento daqueles que apresentam maior eficiência produtiva, a corrupção desfigura os papéis alocativo e redistributivo do Estado, lesando sua legitimidade política (Rose-Ackerman, 1999).

A corrupção é apresentada por Croix \& Delavallade (2009) como um dos principais fatores que impedem os países em desenvolvimento de recuperar o atraso em relação aos mais desenvolvidos. Bardhan (1997) e Abed \& Gupta (2002) explicam que a corrupção é um elemento-chave para a incapacidade das sociedades pobres em tirar proveito das oportunidades de desenvolvimento.

Nesse sentido, o controle da corrupção é um dos assuntos que se encontram no topo da agenda das organizações internacionais relacionadas com desenvolvimento econômico. Por exemplo, o Banco Mundial e a Organização para Cooperação e Desenvolvimento Econômico (OCDE) apoiam o desenvolvimento de programas anticorrupção no intuito de melhorar a capacidade da gestão pública, promover o desenvolvimento econômico e a luta contra a pobreza (Croix \& Delavallade, 2009).

Para Anderson \& Tverdova (2003), a corrupção reduz a legitimidade do governo perante a sociedade, podendo gerar um cenário de instabilidade política. Del Monte \& Papagni (2007) acrescentam que a qualidade das instituições políticas e sociais desempenha um papel importante nos esforços para controlar a corrupção.

Os estudos sobre a temática demonstram que a corrupção causa: distorções orçamentárias (Croix \& Delavallade, 2009), diminui os gatos do governo com educação (Mauro, 1998; Tanzi, 1998; Delavallade, 2006), reduz o crescimento econômico (Mo 2001; Croix \& Delavallade, 2009), aumenta o custo do serviço da dívida (Depken \& Lafountaine,2006) e provoca ineficiência na alocação de recursos na área de saúde e educação (Lopes \& Toyoshima, 2013).

No Brasil, o fenômeno da corrupção perpassa as diferentes esferas administrativas nos três níveis de governo. Na mídia, é recorrente a eclosão de escândalos de corrupção envolvendo membros, do executivo, do legislativo ou judiciário, na União, estados ou municípios. Escândalos como Sanguessugas, Mensalinho, Mensalão, Operação Lava-Jato, entre outros, foram recorrentes nos noticiários e desvendaram diversos esquemas de corrupção nas três esferas de governo e com a associação de indivíduos de diversos partidos (Madeira \& Geliski, 2020; Feres Júnior \& Sassara, 2016; Praça, 2011)

Sodré \& Alves (2010) destacam que a publicação do Índice de Percepção da Corrupção da organização Transparência Internacional, que avalia a percepção de empresários e analistas de governo quanto à presença e o nível de corrupção em diversos países, evidencia que o Brasil está atrás de países com igual ou pior indicadores de atividade econômica, como Uruguai, Colômbia, Cuba, Costa Rica e África do Sul.

Apesar da crescente exposição de casos de corrupção por meio da mídia ou das tecnologias da informação, a mensuração da corrupção ainda é difícil de ser realizada. No âmbito nacional existem índices que mensuram a percepção da corrupção em diversos países. Como exemplo, cita-se os disponibilizados pela Transparency International, World Bank e World Business Environment Survey. No entanto são poucas as informações disponíveis que permitam mensurar a corrupção em governos subnacionais.

Melo (2010) defende que detectar a manifestação da corrupção é uma tarefa complexa, principalmente em governos subnacionais, pois depende de uma logística que abrange desde sistemas de controle social até instrumentos de accountability institucional. $\mathrm{O}$ autor ainda destaca que, na maioria dos crimes, há sempre uma vítima ou indivíduo envolvido que se propõe a denunciar. Em contraponto, nos casos de corrupção, o corrupto e corruptor buscam a todo custo minimizar a possibilidade de descoberta de seus atos, o que dificulta a apuração desse fenômeno e, consequentemente, sua mensuração.

Diante da dificuldade de mensuração da corrupção e dos seus efeitos negativos sobre a gestão pública, busca-se, com o presente artigo, medir o nível de corrupção nos estados brasileiros, por meio da construção de um índice estadual de corrupção (ICE), e verificar possíveis relações entre investimentos públicos, saúde, educação, endividamento e o índice de corrupção.

$\mathrm{Na}$ literatura nacional observam-se alguns esforços para a mensuração da corrupção em nível estadual. Boll (2010) e Carraro, Machado, Conever \& Boll (2015) utilizaram-se de informações do Cadastro de Contas Irregulares do Tribunal de Contas da União (Cadirreg) para construir indicadores sobre a corrupção. Já Lopes \& Toyoshima (2013) utilizaram como proxy de corrupção o número de matérias sobre corrupção publicadas nos principais jornais e revistas em cada unidade federativa do Brasil. Melo, Sampaio e Oliveira (2015), ao analisarem as relações entre empreendedorismo e corrupção, fizeram uso do indicador de Boll (2010) para mensurar a corrupção. Buscando contribuir com o avanço na literatura sobre o tema, este estudo considerou os dados disponíveis sobre processos e condenações judiciais relacionados a casos de corrupção para a construção de um Índice de Corrupção Estadual, abrangendo delitos cometidos por atores governamentais, bem como por pessoas físicas e jurídicas. Dessa forma, é possível mensurar a corrupção de uma forma distinta e ainda comparar os resultados com as evidências encontradas pelos estudos anteriores. 
O estudo se justifica na medida em que a corrupção tem efeitos negativos sobre a gestão pública, pois gera a evasão de receitas e a ineficiência na alocação de recursos, principalmente nas áreas de educação e saúde (Kaufmann, Kraay \& Mrastruzzi, 2011; Lopes \& Toyoshima, 2013). Dessa forma, conhecer o nível de corrupção dos estados brasileiros pode contribuir para identificar onde esse fenômeno tem maior e menor incidência, quais são os possíveis casos exemplares quais práticas de gestão contribuem para o controle da corrupção e, consequentemente, obter uma melhor aplicação do recurso público.

\section{REVISÃO DE LITERATURA}

Esta seção é voltada para a construção teórica dos principais conceitos relacionados com a corrupção, bem como a relação entre corrupção e elementos da gestão pública, como investimento, saúde e educação.

\subsection{Corrupção: conceitos e causas}

A corrupção é considerada um fenômeno que afeta todos os países, independentemente de seu nível de desenvolvimento. Conforme defendido por Treisman (2000), a corrupção tem sua causa associada com aspectos históricos, culturais, tradições, a instituições políticas e a políticas governamentais e níveis de desenvolvimento econômico.

Em trabalho seminal sobre o tema, Brooks (1909) apresenta que a corrupção, de modo geral, está associada a atividades como fraude, suborno, propina, saques e/ou desvios de verbas. O autor define a corrupção como o mau desempenho proposital ou o desmazelo com relação à obrigação institucionalmente reconhecida, ou o exercício de forma indiscriminada do poder, com intuito de beneficiamento próprio.

Nessa mesma perspectiva, Tanzi (1998) considera que a corrupção é caracterizada pela quebra de um código de conduta moral, social ou regra administrativa, e, para que tal quebra aconteça, também é necessário que os agentes envolvidos obtenham algum tipo de benefício para si próprio, ou seja, uma compensação pelo ato de corrupção, mais conhecida como propina.

Conforme destaca Lopes \& Toyoshima (2013), a corrupção é normalmente entendida como o uso de bens, serviços e do poder público para o benefício privado, podendo assumir diversas formas, como pagamento de propinas, fraudes em licitações, desvios de verbas e compra de votos.

Para Boll (2010), há uma diferenciação entre os tipos de corrupção, sendo que a governamental é aquela que acontece quando os agentes envolvidos rompem os códigos de conduta que regulamentam sua atuação no setor público para obterem de forma ilícita sua recompensa financeira com o desvio de recursos públicos.

Sodré \& Alves (2010) alertam que é preciso distinguir corrupção de um conceito correlato, porém não idêntico, que é o de irregularidades de gestão, isto é, a falta de observância às legislações pertinentes e das boas práticas administrativas no setor público. Os problemas relacionados com a corrupção podem ser causados por disfunções gerenciais ou atos propriamente fraudulentos contra o erário público, ambos implicam em prejuízo aos recursos públicos, mas diferenciam-se em relação à intencionalidade dos agentes. De qualquer forma, há que se perceber que a segunda hipótese raramente ocorre sem a primeira.

Ao lançar olhar sobre os estudos que abordam a temática da corrupção no Brasil, Filgueiras (2009) considera que estes são recentes, realizados a partir de abordagens comparativas e institucionalistas, sem a pretensão de uma teoria geral de cunho interpretativo.

Para Avritzer \& Filgueiras (2011), no Brasil, a organização do sistema político, a organização do Estado e a organização das formas de controle sobre o sistema administrativoestatal são os principais componentes delineadores da corrupção e que a tornam um fenômeno fortemente contencioso. Sendo assim, a espera pela ruptura cultural e institucional com o passado, como pressupõe a interpretação pelo conceito de patrimonialismo, não se apresenta como a melhor solução para se pensar o problema da corrupção no Brasil.

Dessa forma, a presença maciça da corrupção no debate público causa a deterioração da confiança dos cidadãos nas instituições políticas e a construção de um processo de crescente deslegitimação das instituições tradicionais de representação política, entre elas, os partidos políticos e os legislativos. Como resultado, em meio ao processo de expansão da visibilidade da corrupção, o processo de democratização no Brasil tem promovido cada vez mais a participação da sociedade civil na formulação e no controle das políticas públicas (Filgueiras, 2009).

Conforme destacam Filgueiras \& Aranha (2011), o Brasil vive um paradoxo entre a inovação de práticas gerenciais na administração pública e a permanência da corrupção, no que se configura como uma agenda negativa da esfera pública, marcada por uma sucessão de escândalos políticos que causam danos à legitimidade do Estado.

As discussões científicas sobre a corrupção no setor público têm como um dos pontos centrais suas causas. Melo (2010) acredita que, nesse aspecto, existe um consenso que se trata de um fenômeno multicausal. Fisman \& Miguel (2007) defendem que, apesar de amplamente discutidas, as causas da corrupção ainda são mal compreendidas.

A literatura aponta que a corrupção pode ser explicada por diversos fatores, como o poder discricionário dos agentes envolvidos, recursos existentes, cálculo racional atrelado a baixos salários, excesso de regulamentação, baixos níveis de accountability, fragmentação étnica e cultural, qualidade das instituições, contexto democrático, competitividade econômica, nível de tributação, tamanho do governo e aspectos históricos (Melo, 2010). 
Aranha (2013) destac,a quanto aos aspectos étnicos, culturais e históricos, que no Brasil é necessária uma análise cautelosa para não generalizar as interpretações como as de Sérgio Buarque de Holanda (1995), que defende a ideia da cultura brasileira como fortemente patrimonialista, e de Roberto DaMatta (1997), que aponta o traço da "malandragem" na ética individual e nas relações sociais do brasileiro. Essas interpretações geram a ideia que o indivíduo brasileiro já possui uma predisposição à corrupção.

Nesse sentido, Filgueiras (2009, pg. 393) defende que "o caráter do brasileiro, como muitos intérpretes procuram delimitar, termina por estabelecer uma armadilha analítica e conceitual, incapaz de perceber que dicotomias mais obscurecem nossas imoralidades do que propriamente as esclarecem".

Ademais, Aranha (2013) considera que analisar o fenômeno da corrupção exige uma abordagem que inclua a apreciação dos valores e concepções morais dos cidadãos de cada coletividade. Isto significa que compreender a corrupção passa pelo entendimento da configuração dos aspectos normativos que fundamentam a vida em sociedade.

Seguindo uma vertente mais econômica, tem-se que a corrupção está condicionada ao modo como as instituições tornam possível a ação discricionária dos atores políticos, ou seja, permitem o uso de recursos públicos para a satisfação de interesses privados. É evidente, nessa abordagem, a necessidade de reformas institucionais para o controle da corrupção (Rose-Ackerman, 1999; Aranha, 2013).

No entanto, independente da causa, para que ocorra a corrupção é necessária à existência de um ambiente propício. Nas, Price \& Weber (1986) consideram que esse ambiente vai depender de características pessoais e estruturais, de forma que as características pessoais se associam fortemente a desvios de caráter, como a cobiça ou a fraqueza frente às tentações, ou seja, a ausência de ética. Já a estrutura é determinada por três fatores: o ambiente burocrático ou organizacional, a qualidade da participação social, e a congruência entre o sistema legal e as demandas sociais.

\subsection{Os efeitos da corrupção na gestão pública}

A corrupção tem uma série de efeitos indesejáveis para a gestão pública e atinge, principalmente, a área social (Abed \& Gupta, 2002). Tal fato levou ao desenvolvimento de diversos estudos sobre essa temática (Mauro, 1998; Treisman, 2000; Mo, 2001; Del Monte \& Papagni, 2001; Svensson, 2005; Serra, 2006; Depken \& Lafountaine, 2006; Pellegrini \& Gerlagh, 2008; Croix \& Delavallade, 2009; Lopes \& Toyoshima, 2013; Witvliet, Kunst, Arah \& Stronks, 2013).

O foco central desses estudos é entender quais são os efeitos da corrupção sobre a economia e a gestão pública. Mauro (1998) encontrou que a corrupção está negativamente correlacionada com os gastos de governos com educação, indicando ser essa uma área de desvio de recursos pelos corruptores. O autor aponta que o resultado é preocupante, porque a educação é um dos condicionantes do crescimento econômico.

Os resultados de Treisman (2000) evidenciam que países com maiores níveis de desenvolvimento, maiores volumes de exportação e com maior incidência da religião protestante são menos corruptos. Já Del Monte \& Papagni (2001) demonstram que a corrupção causa ineficiência nos investimentos públicos, inibindo o crescimento econômico.

Mo (2001) demonstrou que a corrupção reduz o crescimento econômico, principalmente porque provoca instabilidade política, redução do nível de capital humano e diminui o peso do investimento do setor privado. Nessa mesma linha de raciocínio econômico, Serra (2006), ao analisar os determinantes, verificou que a corrupção é menor nos países mais ricos, onde as instituições democráticas foram preservadas por um período contínuo de tempo e a população é principalmente protestante. A corrupção está cada vez maior onde a instabilidade política é um grande problema.

Del Monte \& Papagni (2007) mostram que, na Itália, a despesa pública em bens e serviços de consumo parece ser uma causa importante da corrupção. Além disso, observou-se um efeito negativo sobre o crescimento e um aumento do custo da corrupção para a comunidade empresarial. A presença crescente de organizações voluntárias aparentemente reduz a corrupção.

Nas análises de Svensson (2005) o que caracteriza os países com alta corrupção são economias em desenvolvimento ou em transição. Surpreendentemente, muitos são governados, ou foram recentemente governados, pelos governos socialistas, têm baixos níveis de rendimento e são consideradas economias fechadas de uma forma geral.

Os efeitos fiscais da corrupção foram analisados por Depken \& Lafountaine (2006), que demonstram que, após controlar variáveis de influência econômica sobre ratings de títulos, há evidências que, em governos mais corruptos, as classificações de ratings são mais baixas, o que implica que os contribuintes enfrentam uma externalidade negativa mediante o pagamento de um prêmio para a dívida. Dessa forma, a corrupção aumenta os custos da dívida pública e, consequentemente, o endividamento governamental.

Pellegrini \& Gerlagh (2008), ao testarem as principais hipóteses presentes na literatura, encontraram que o sistema de comom-law, ou um passado como uma colônia britânica, não pode prever a corrupção. Os resultados apoiam teorias culturais sobre as causas da corrupção, e sugerem que uma exposição de médio e longo prazo para a democracia ininterrupta está associada com níveis de corrupção mais baixos, enquanto o volume de negociações políticas, devido à necessidade de acordos para governabilidade, e a existência de grande número de partidos tende a elevar a corrupção. Os resultados também sugerem que o fortalecimento e a difusão da mídia podem ajudar a reduzir os níveis de corrupção. 
Croix \& Delavallade (2009) demonstram que o investimento público pode ser distorcido em favor de tipos específicos de gastos para os quais o rent-seeking é mais fácil e melhor escondido pelos atores de corrupção. Os autores observam que, nos países em desenvolvimento, os investimentos são mais distorcidos nas áreas de habitação, capital físico, saúde e educação. Lopes \& Toyoshima (2013) corroboram esses resultados ao demonstrarem que a corrupção causa ineficiência na alocação de recursos públicos nas políticas das áreas de saúde e educação.

Witvliet et al. (2013) mostram associações positivas entre saúde precária e corrupção percebida, sendo esse fato evidente em todos os grupos socioeconômicos, com a associação um pouco mais positiva entre as pessoas com menor escolaridade do que entre pessoas mais escolarizadas. Dessa forma, os autores demonstram que a educação é mais uma área de incidência desse fenômeno.

Como se pode observar, na literatura levantada, de forma geral, os efeitos da corrupção são mais evidentes sobre o crescimento econômico e a área social como saúde e educação. Destaca-se que esse é um círculo vicioso à medida que o desenvolvimento é atrelado ao bom desempenho do governo, principalmente nas áreas mais afetadas pela corrupção.

\section{PROCEDIMENTOS METODOLÓGICOS}

No presente artigo foi realizada uma abordagem quantitativa com delineamento dos dados em painel referente ao período de 2010 a 2012. Quanto aos objetivos, caracteriza-se como uma pesquisa descritiva, pois se busca descrever e analisar a incidência da corrupção nos estados brasileiros e sua relação com investimento público, despesas com saúde e educação, e endividamento.

\subsection{A construção do Índice de Corrupção Estadual (ICE)}

No intuito de identificar os fatores que permitam explicar o nível de corrupção nos estados brasileiros, o modelo de análise fatorial exploratória foi utilizado por se tratar de uma técnica de análise multivariada apropriada para essa finalidade, uma vez que os fatores e escores fatoriais obtidos serão utilizados para a mensuração e construção do Índice Estadual de Corrupção (ICE).

Segundo Mingoti (2007), as novas variáveis identificadas na análise fatorial são denominadas de fatores ou variáveis latentes, e o modelo ortogonal apresenta a seguinte expressão analítica:

$$
X_{p x 1}=A_{p x r} \cdot F_{r x 1}+\varepsilon_{p x 1}
$$

Em que: $X$ é o vetor de variáveis originais; $F$ é o vetor de fatores comuns; $A$ é a matriz de cargas fatoriais; $\varepsilon$ é o vetor de erros aleatórios (fatores específicos mais erro); e $r$ (número de fatores) $<p$ (número de variáveis).

Os fatores são estimados mediante combinação linear das variáveis originais, conforme apresentado nas equações 2 e 3 :

$$
\begin{gathered}
F_{j}=\omega_{j 1} \cdot X_{1}+\omega_{j 2} \cdot X_{2}+\omega_{j 3} \cdot X_{3}+\cdots+\omega_{j i} \cdot X_{i} \\
F_{j}=\sum_{i=1}^{n} \omega_{j i} \cdot X_{i}
\end{gathered}
$$

Em que: $F_{j}$ são os fatores comuns não relacionados, $\omega_{i i}$ são os coeficientes dos escores fatoriais e $X_{i}$ são as variáveis originais envolvidas no estudo, sendo que o escore fatorial é um número resultante da multiplicação dos coeficientes $\omega_{i i}$ pelo valor das variáveis originais (Hair, Black, Babin, \& Anderson, 2009).

Para estimação do modelo de análise fatorial, foi utilizado o método de componentes principais. Mingoti (2007) destaca que esse método não requer informações ou suposições sobre a distribuição normal multivariada dos dados. No intuito de facilitar a interpretação e análise dos fatores, empregou-se o método Varimax de rotação ortogonal.

As variáveis latentes identificadas permitem determinar as relações quantitativas ao agrupar aquelas que apresentam padrão semelhante, indicando os fatores explicativos dos níveis de corrupção estadual. Além disso, os escores fatoriais foram empregados na construção de um índice, conforme é explicado a seguir.

Para a construção do índice, inicialmente, aplicou-se a análise fatorial para a obtenção dos fatores e escores fatoriais. Os procedimentos adotados na elaboração do ICE tiveram como referência os procedimentos tomados por Lemos (2001), Cunha, Lima, Gomes \& Braga (2008) e Ferreira (2015). 
O ICE representa uma proxy que mensura o nível de corrupção dos estados brasileiros. Esse índice permitirá determinar, por intermédio da hierarquização, os estados com maior ou menor incidência do fenômeno, permitindo a identificação de práticas de seu controle na administração pública.

Antes de agrupar as variáveis no índice, é necessário colocar os valores dos escores fatoriais no primeiro quadrante, pois, conforme apresenta Lemos (2001), essa transformação é necessária para evitar que altos escores fatoriais negativos elevem a magnitude dos índices associados a essas observações. Para isso, procede-se à seguinte transformação:

$$
F_{i j}=\frac{\left(F-F_{\min }\right)}{\left(F_{\max }-F_{\min }\right)}
$$

Em que: $F_{\min }$ e $F_{\max }$ são os valores máximo e mínimo observados para os escores fatoriais associados aos municípios mineiros.

Obtidos os valores padronizados dos fatores, para a elaboração do ICE, os fatores foram agregados de acordo com a equação apresentada por Cunha et al. (2008) e Ferreira (2015):

$$
I C E_{i}=\sum_{j=1}^{p} \frac{\lambda_{j}}{\Sigma \lambda_{j}} F_{j i}^{*}
$$

Em que: IPCS é o índice do i-ésimo município, $j$ é a j-ésima raiz característica, $p$ é o número de fatores extraídos na análise, $F_{j i}^{*}$ é o j-ésimo escore fatorial do i-ésimo município e $\Sigma \lambda_{j}$ é o somatório das raízes características referentes aos $\mathrm{p}$ fatores extraídos. A participação relativa do fator $j$ na explicação da variância total captada pelos $p$ fatores extraídos é indicada por $\frac{\lambda_{j}}{\Sigma \lambda_{j}}$.
Os valores do índice foram, então, reparametrizados para assumirem valores entre 0 e 100 .

\subsection{Relações entre corrupção e investimento público, educação, saúde e endividamento.}

No intuito de estabelecer possíveis relações entre o nível de corrupção dos estados e investimento público, educação, saúde e endividamento, conforme indicado pela teoria, foi utilizado o teste de KrusKal-Wallis.

Esse teste, segundo apresentam Fávero et al. (2009), verifica a probabilidade de que K amostras independentes sejam provenientes de uma mesma população, sendo o teste sugerido para os casos em que a amostra é pequena e/ ou quando as suposições da análise de variância são violadas. No caso deste estudo, o teste indicara se há diferença entre grupos classificados de acordo com o nível de corrupção em relação ao investimento público, despesas com saúde e educação, endividamento e taxa de alfabetização.

\subsection{Variáveis empregadas}

Para identificar os fatores explicativos que contribuem para a mensuração do nível de corrupção estadual, este estudo considerou os dados disponíveis sobre processos e condenações judiciais relacionados a casos de corrupção. Essas variáveis foram coletadas nos bancos de dados disponibilizados pelo Ministério Público Federal (MPF) e no Portal da Transparência. Foram realizadas buscas no Portal da Transparência com relação aos processos relacionados à corrupção. Em caso de dúvidas, eram consultadas informações no site do MPF. Já na aba de sanções do Portal da Transparência foram consultadas as informações sobre as pessoas expulsas e pessoas inidôneas. As informações coletadas eram todas desagregadas, e classificadas e organizadas por estado com auxílio do software MS Excel. As variáveis selecionadas são listadas a seguir:

X1 = Número de processos penais;

X2 = Número de processos administrativos;

X3 = Número de pessoas expulsas da administração pública federal;

X4 = Número de pessoas jurídicas inidôneas;

X5 = Número de pessoas físicas inidôneas;

Para testar as hipóteses dos efeitos da corrupção sobre a gestão pública foram escolhidas variáveis que representassem proxys de educação, saúde, investimento público e endividamento. Tais variáveis foram coletadas nos bancos de dados do Ipeadata e do IBGE. As variáveis selecionadas são apresentadas a seguir: 
X6 = Despesas com educação em relação ao PIB estatal;

X7 = Despesa com saúde em relação ao PIB estatal;

X8 = Taxa de alfabetização;

X9 = Investimento público (despesa de capital) em relação ao PIB estatal;

X10 = Dívida mobiliária em relação ao PIB estatal (A dívida mobiliária refere-se à dívida constituída a partir da emissão de títulos públicos).

\section{4. Área de estudo e tratamento dos dados}

Este estudo abrange os 26 estados brasileiros e o Distrito Federal, com vistas a viabilizar uma análise de escopo estadual. Buscando o tratamento e a análise dos dados coletados, utilizou-se a planilha eletrônica Excel e os softwares Statistical Package for Social Sciences (SPSS), em sua versão 24.0, e Stata, em sua versão 14.0.

\section{APRESENTAÇÃO E ANÁLISE DOS DADOS}

\subsection{Análise do Índice de Corrupção Estadual (ICE)}

Por meio dos resultados da análise fatorial, pôde-se verificar que os testes de adequação da amostra se apresentaram satisfatórios. O teste de esfericidade de Bartlett foi significativo estatisticamente a menos que $1 \%$, ou seja, rejeita-se a hipótese nula de que a matriz de correlações é igual a uma matriz identidade. Já o critério KMO apresentou resultado de 0,590, superior ao patamar crítico aceitável de 0,50, conforme indicado por Hair et al. (2009).

A utilização da análise fatorial com decomposição em componentes principais resultou na extração de dois fatores, considerando o critério de Kaiser, de raízes características $(\lambda)$ maiores que 1, como pode ser verificado na Tabela 1.

Tabela 1

Número de Fatores e Porcentagem de Explicação da Variância

\begin{tabular}{cccc}
\hline Fator & Raiz Característica & Inicial (\%) & Rotativa (\%) \\
\hline 1 & 1,901 & 47,096 & 38,02 \\
2 & 1,732 & 25,561 & 34,64 \\
Total & - & 72,657 & 72,66 \\
\hline
\end{tabular}

Fonte: Elaboração própria.

Os fatores, após a rotação ortogonal Varimax, apresentaram, respectivamente, uma explicação de 38,02\% e de $34,64 \%$ da variância, sendo que, em conjunto, esses dois fatores explicam $72,66 \%$ da variância das variáveis originais. Na Tabela 2 são apresentados os resultados das cargas fatoriais rotacionadas e das comunalidades.

As comunalidades demonstram a proporção da variância para cada variável estudada que é explicada pelos dois fatores extraídos, ou seja, as comunalidades indicam as variáveis consideradas mais representativas na construção dos componentes. No caso deste estudo, as variáveis mais representativas foram o número de processos penais e o número de processos administrativos.

Normalmente, o valor mínimo aceitável para a comunalidade é de 0,50 . Sendo assim, os resultados demonstram que todas as variáveis apresentaram valores acima do aceitável, indicando a continuidade da análise.

Tabela 2

Cargas Fatoriais Rotacionadas e Comunalidades

\begin{tabular}{c|c|cc}
\hline \multirow{2}{*}{ Variáveis } & \multicolumn{2}{c}{ Cargas Fatoriais } & \multirow{2}{*}{ Comunalidades } \\
\cline { 2 - 3 } & F1 & F2 & \\
\hline Processo penal & 0,941 & 0,117 & 0,903 \\
Processo de improbidade & 0,917 & 0,131 & 0,854 \\
Pessoas físicas inidôneas & 0,314 & 0,788 & 0,640 \\
Pessoas jurídicas inidôneas & $-0,135$ & 0,744 & 0,612 \\
Pessoas expulsas & 0,241 & 0,725 & 0,624 \\
\hline
\end{tabular}

Fonte: Elaboração própria. 
Além das comunalidades, precisam ser analisadas as cargas fatoriais de cada variável em relação aos componentes extraídos. Os resultados apresentados na Tabela 2 evidenciam que foi atendido o critério apresentado por Mingoti (2007) de uma mesma variável não contribuir acima de 0,40 para a construção dos dois fatores. Esse limite é indicado para evitar problema de indeterminação da relação entre variáveis e fatores. Em face disso, foi possível identificar, sem maiores dificuldades, quais variáveis se relacionam com quais fatores.

Determinados os fatores retidos e a sua validade procurou-se discutir cada um deles:

- Fator 1 = Processos judiciais: Refere-se aos aspectos de apuração de fatos que tem indícios de corrupção cometidos por atores públicos ou privados em seu relacionamento com o setor público.

- $\quad$ Fator 2 = Sanções: Relaciona-se com as penalidades aplicadas a atores públicos, pessoas físicas ou jurídicas que as impedem de atuar ou efetuar transações com o setor público devido ao cometimento de atos corruptos.

Com a finalidade de hierarquizar os estados brasileiros em termos de nível de corrupção, foi construído o ICE. Ao considerar conjuntamente os fatores Processos judiciais e Sanções em um índice, foi possível classificar os estados brasileiros, demonstrando aqueles em que há maior ou menor incidência de corrupção.

Apesar da possível fragilidade no índice calculado, devido ao fato da possibilidade de governos com maior incidência de corrupção não abrirem processos judiciais ou aplicarem sanções a seus atores, e vice-versa, diante das informações disponíveis, o autor considera que essa é a melhor forma de mensuração possível da corrupção nos estados brasileiros, porque as variáveis utilizadas na construção do índice abrangem tantos delitos cometidos por atores governamentais quanto aqueles cometidos por pessoas físicas e pessoas jurídicas.

O índice calculado mediante os fatores é uma proxy, que representa o nível de corrupção de cada estado brasileiro. Sob essa ótica, torna-se importante analisar a estatística descritiva do ICE, sendo que, para facilitar a interpretação dos dados, o índice foi reparametrizado a partir do maior valor. A amplitude dos dados demonstra o valor do desvio padrão em relação à média, conforme pode ser observado na Tabela 3, e evidenciam discrepâncias com relação ao nível de corrupção entre os estados brasileiros.

Tabela 3

Estatística Descritiva

\begin{tabular}{cccccccccc}
\hline & Média & Mediana & $\begin{array}{c}\text { Desvio } \\
\text { Padrão }\end{array}$ & Variância & Assimetria & Curtose & Amplitude & Mínimo & Máximo \\
\hline ICE & 43,75 & 36,45 & 24,23 & 587,24 & 0.68 & $-0,72$ & 89,62 & 10,38 & 100,00 \\
\hline
\end{tabular}

Fonte: Elaboração própria.

$\mathrm{Na}$ análise realizada, o estado que apresentou o menor nível de corrupção foi o do Paraná, com o valor do índice referente ao ano de 2012. Já o maior nível de corrupção, no ano de 2012, foi observado no Pará.

Para permitir a melhor visualização da incidência da corrupção em todos os estados de forma conjunta, a Figura 1 apresenta a distribuição do índice de corrupção para o ano de 2010.

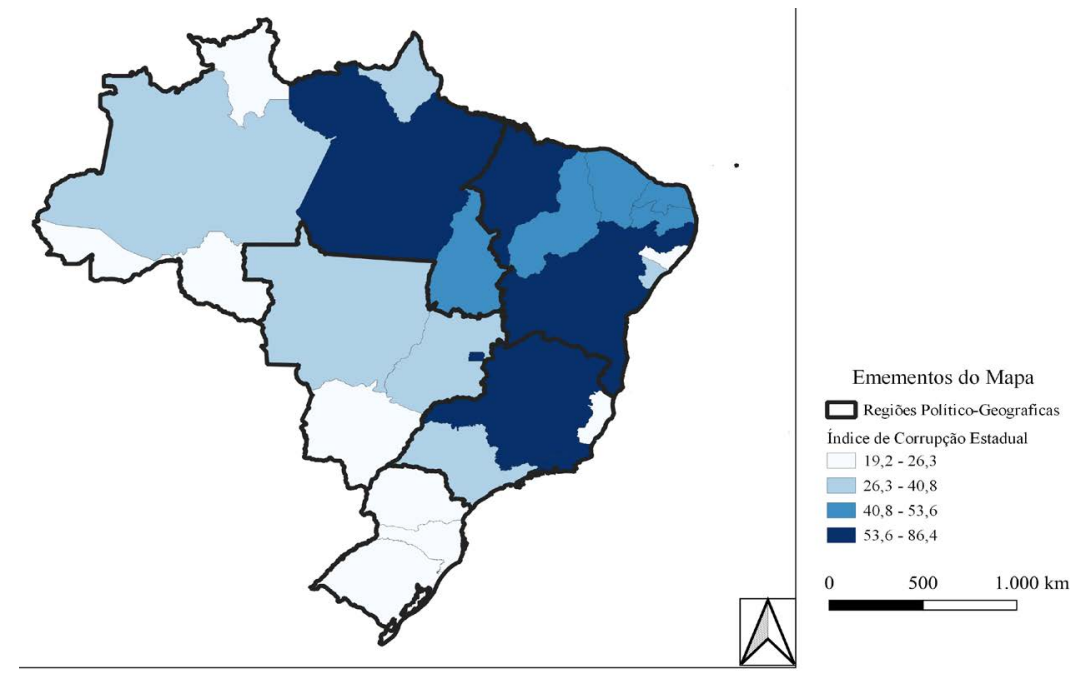

Figura 1. Distribuição do índice de corrupção em 2010.

Fonte: Elaboração própria. 
Os estados que apresentaram maior índice de corrupção, em 2010, foram Pernambuco $(86,42)$, Minas Gerais $(83,51)$ e Distrito Federal $(83,46)$. Já os menores valores do índice são observados para Rio Grande do Sul $(19,21)$, Santa Catarina $(19,47)$ e Roraima $(19,75)$.

Na Figura 2 é apresentada a distribuição do ICE para o ano de 2011.

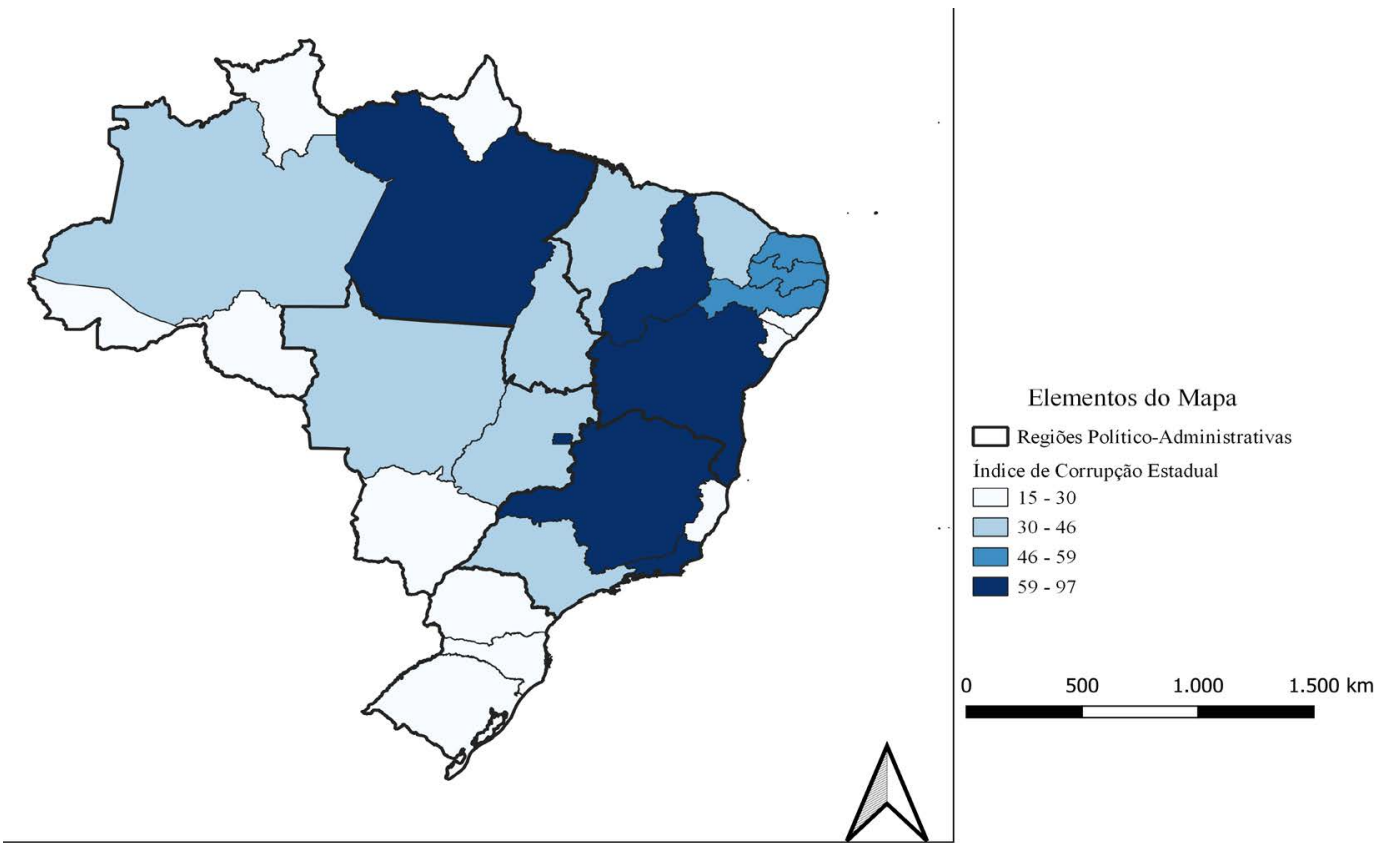

Figura 2: Distribuição do índice de corrupção em 2011.

Fonte: Elaboração própria.

No ano de 2011, observa-se que os menores valores do índice de corrupção são observados nos estados de Rio Grande do Sul $(14,58)$, Paraná $(15,15)$ e Santa Catarina $(16,23)$. Já a maior incidência de corrupção é encontrada nos estados do Pará $(97,09)$, Bahia $(83,65)$ e Minas Gerais $(83,59)$.

O panorama da corrupção nos estados brasileiros no ano de 2012 são apresentados na Figura 3.

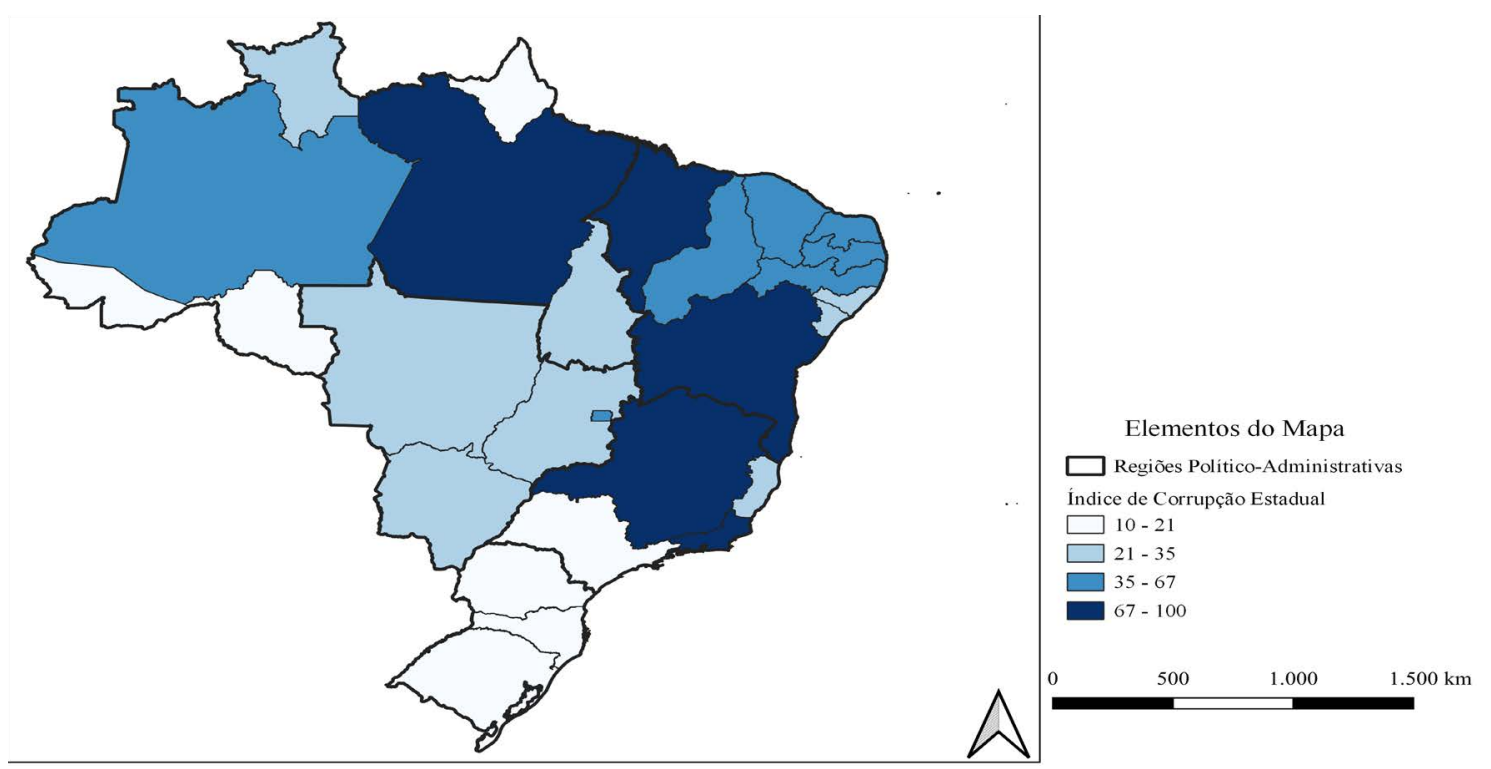

Figura 3: Distribuição do índice de corrupção em 2012.

Fonte: Elaboração própria. 
No ano de 2012, o estado do Pará apresenta novamente o maior valor do índice de corrupção $(100,00)$, seguido pelos estados do Maranhão $(89,74)$ e da Bahia $(88,69)$. Os menores valores do índice de corrupção são observados para os estados do Paraná $(10,68)$, Rio Grande do Sul $(11,82)$ e Santa Catarina $(13,52)$.

No estudo desenvolvido por Carraro et al. (2015) apresenta-se como resultado que os estados brasileiros com maior incidência de corrupção são: Bahia, São Paulo e Maranhão. Corroborando em partes com o estudo do autor, o estado da Bahia apresentou valores altos e crescentes para o índice de corrupção, sendo de 76,37 em 2010 e de 88,69 em 2012, figurando entre os estados mais corruptos. O estado do Maranhão apresenta variação nos valores dos índices de corrupção, com 69,50 para o ano de 2010, 45,87 em 2011 e 89,74 em 2012, ano em que esteve entre os estados mais corruptos do país. Já o estado de São Paulo, apresentou valores medianos e decrescentes para o nível de corrupção com valor de 40,79 em 2010 e 20,63 em 2012, não figurando entre os estados mais corruptos do país. Destaca-se que, neste estudo, o Pará apresenta níveis altos e crescentes de corrupção para os três anos de análise, com valor de 75,32 no 2010 e de 100,00 no ano de 2012.

Seguindo o mesmo sentido de comparação, Carraro et al. (2015) encontraram que os estados brasileiros com menor ocorrência de corrupção são Acre, Rondônia e Santa Catarina. Confirmando esse resultado, o estado de Santa Catarina também se encontra entre os com menores níveis de corrupção nos três anos de análise deste estudo. O estado de Rondônia também apresenta valores baixos e decrescentes para o índice de corrupção, com valor de 20,7 em 2010 e de 17,8 em 2012, estando entre os estados menos corruptos. O estado do Acre também apresenta valores baixos e decrescentes no índice de corrupção, indo de 22,06 em 2010 para 18,51 em 2012. Evidencia-se que, neste estudo, o estado do Rio Grade do Sul destaca-se entre os estados com menor incidência de corrupção, apresentando valores baixos e decrescentes para o índice de corrupção, conforme apresentado.

Demonstra-se, na Figura 4, a incidência do fenômeno da corrupção nas regiões geográficas do Brasil no período analisado.

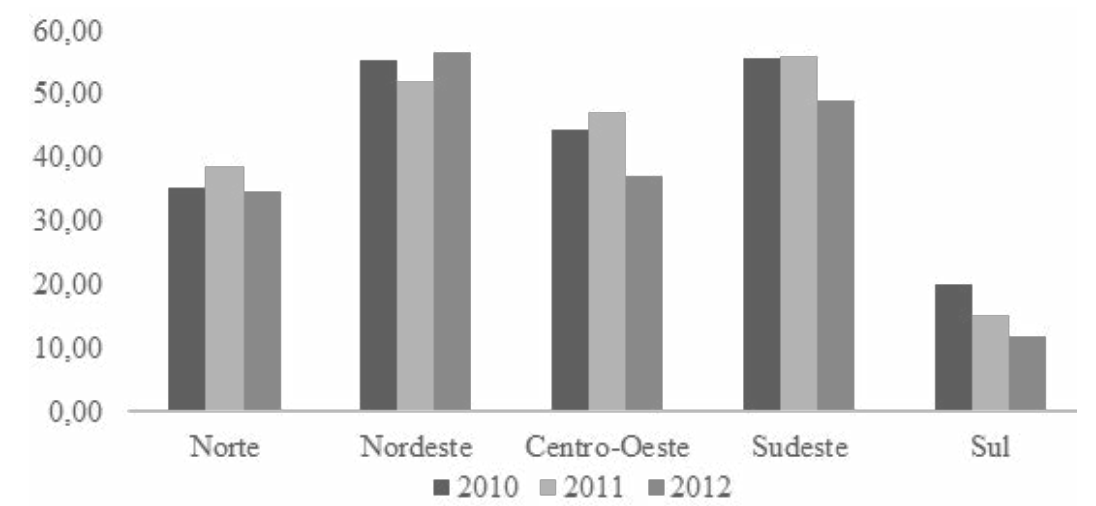

Figura 4: Nível de corrupção nas regiões geográficas brasileiras

Fonte: Elaboração própria.

Pode-se observar que a Região Sul é a que apresenta o menor nível de corrupção para o período analisado, resultado consoante com o de Boll (2010). Além disso, foi a única região com redução continua nos valores do ICE, redução esta apresentada pelos três estados da região no período analisado.

Já os maiores níveis de corrupção são observados nas Regiões Nordeste e Sudeste. Destaca-se que, na Região Sudeste, o valor médio é inflacionado pelos altos níveis de corrupção apresentado pelos estados de Minas Gerais e Rio de Janeiro nos três anos analisados. Na análise temporal, observa-se uma redução no nível de corrupção no ano de 2012 em relação aos anos anteriores, com exceção da Região Nordeste. Tal redução pode ser explicada por ações desenvolvidas pelo poder público, principalmente dos tribunais de contas e do poder judiciário, no intuito de controlar a prática de corrupção no setor público, tais como a instituição de CPIs e operações para apuração e julgamento de desvios de recursos públicos, além da cobrança pelo desenvolvimento dos portais de transparência no país.

Devido às informações apresentadas pela análise descritiva do ICE, realizaram-se dois diferentes testes de normalidade (Kolmogorov-Smirnov e Shapiro-Wilk), que indicaram que o índice não segue uma distribuição do tipo normal. Por isso, para facilitar a compreensão dos diferentes níveis de corrupção nos estados, estratificou-se o índice em quatro a partir dos quartis. A Tabela 4 mostra o nível de cada um dos estratos analisados. 
Tabela 4

Divisão de grupos de acordo com o nível de corrupção

\begin{tabular}{ccc}
\hline Nível de Corrupção & Valores do ICE & $\mathbf{N}^{\circ}$ de Observações \\
\hline Alto & $56,96-100,00$ & 20 \\
Médio-alto & $56,95-36,46$ & 21 \\
Médio-baixo & $26,37-36,45$ & 20 \\
Baixo & $10,38-23,36$ & 20 \\
\hline
\end{tabular}

Fonte: Elaboração própria.

Após estabelecidos os grupos com diferentes níveis de corrupção, procedeu-se ao teste de KrusKal-Wallis, com o intuito de estabelecer relações entre o nível de corrupção e investimento público, despesa com educação e saúde e alfabetização. Os resultados do teste são apresentados na Tabela 5.

Tabela 5

Resultados Teste KrusKal-Wallis

\begin{tabular}{cccccc}
\hline & INV & DESP.EDU & DESP.SAÚ & DIV.PUB & ALF \\
\hline $\mathbf{X}^{2}$ & 3,226 & 6,857 & 2,826 & 7,557 & 12,315 \\
Significância & 0,358 & 0,077 & 0,419 & 0,056 & 0,006 \\
\hline
\end{tabular}

Fonte: Elaboração própria.

Para a variável investimento público, a expectativa teórica, conforme apresentada por Croix \& Delavallade (2009), é que houvesse relação significativa com a corrupção, uma vez que causa distorções no investimento. No entanto o resultado do teste aponta que não se pode fazer essa relação, uma vez que não há diferença entre os grupos em relação ao nível de investimento, ou seja, o investimento público nos estados brasileiros pode não ser objeto alvo de ações corruptas.

Apesar das expectativas teóricas criadas com base no trabalho de Lopes \& Toyoshima (2013) e Witvliet et al. (2013), os resultados do teste também não permitem inferir relações entre corrupção e gastos com saúde, uma vez que também não há diferença entre os grupos com relação a essa variável. Dessa forma, também há indicativos que a corrupção não afeta a área de saúde nos estados brasileiros. No intuito de analisar as relações que apresentaram significância estatística foi elaborada a Figura 2.

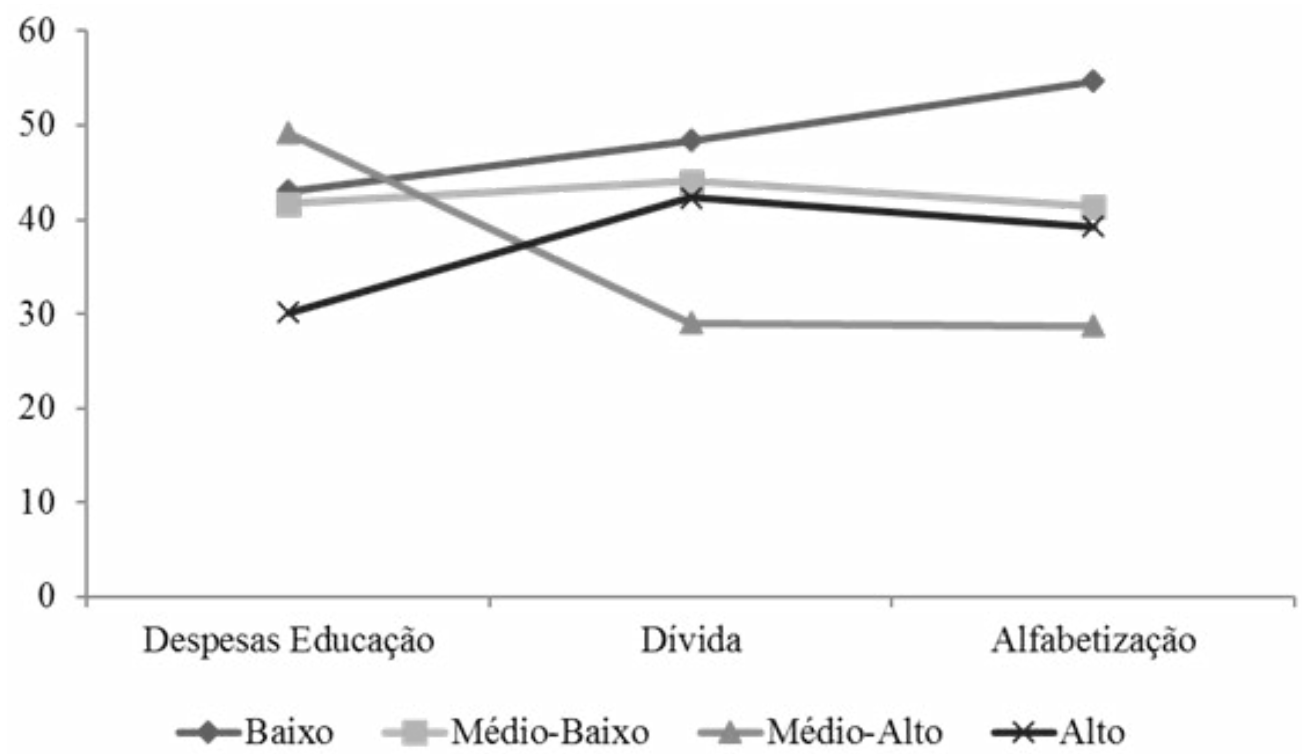

Figura 5. Despesa com educação, dívida pública e alfabetização por grupo de nível de corrupção.

Fonte: Elaboração própria. 
Os resultados do teste apontam para diferença entre os grupos em relação à despesa com educação, indicando a existência de relação entre corrupção e despesa com educação, com nível de significância de $10 \%$. Tal resultado corrobora os achados de Mauro (1998) e Lopes \& Toyoshima (2013). Observa-se que o grupo que apresenta nível de corrupção alto é o que possui menor despesa com educação, demonstrando que a corrupção pode distorcer os investimentos públicos nessa área, conforme indica Croix \& Delavallade (2009). Resultado inverso ao esperado é o encontrado para o grupo com nível médio-alto de corrupção, que apresenta a maior despesa com educação. No entanto, tal resultado pode ser explicado, por exemplo, pelo maior volume de despesas na educação apresentada pelos estados, sem que o recurso seja investido na área, uma consequência da corrupção.

O teste também revelou diferença entre os grupos com relação à dívida pública, o que aponta uma relação significativa entre endividamento e corrupção pelos resultados do teste, com um nível de $10 \%$. No entanto tal evidência contraria as expectativas teóricas apresentadas por Depken \& Lafountaine (2006), pois os estados com maior nível de corrupção apresentam menor endividamento.

O nível de alfabetização da população apresentou relação com o nível de corrupção, com um nível de significância estatística de 1\%. Os resultados corroboram os achados de Mauro (1998), Lopes \& Toyoshima (2013) e Croix \& Delavallade (2009), pois é possível observar que os estados com menor nível de corrupção têm maior percentual da população alfabetizada, indicando uma preocupação maior com a área de educação. Além disso, conforme apresentam Lindstedt \& Naurin (2010), a educação é um elemento essencial para criar condições para o controle social sobre a gestão pública, que tem o poder de inibir a corrupção.

\section{CONSIDERAÇÕES FINAIS}

A mensuração do nível de corrupção dos estados brasileiros revelou que nas regiões Nordeste e Sudeste do país são observados os maiores índices de corrupção. A região Sul apresentou os menores níveis de corrupção para o período analisado, indicando que a análise das práticas de gestão dos estados que compõem a região Paraná, Santa Catarina e Rio Grande do Sul podem indicar casos exemplares sobre medidas de controle da corrupção. Os estados de Rondônia e Acre, localizados na Região Norte, também podem ser considerados casos exemplares nesse sentido, dado que apresentaram valores baixos e decrescentes para o índice de corrupção estadual (ICE) no período de análise.

O ICE desenvolvido neste estudo pode servir como uma medida do nível de corrupção nos estados brasileiros. Pode ainda servir como parâmetro de em quais locais ações de combate à corrupção devem ter mais ênfase, bem como quais estados devem ter suas ações tomadas como possíveis casos exemplares. O índice pode ser aprimorado, em estudos futuros, com a abrangência de um período de tempo maior, o que permitiria um acompanhamento ao longo dos anos, ou, ainda, com a incorporação de novas variáveis indicativas de corrupção em sua composição.

A análise da relação entre a corrupção e o investimento público e as despesas de saúde indicam que essas são possíveis áreas não afetadas pelo fenômeno. No caso da saúde, o resultado pode estar relacionado com os conselhos gestores da área, que são reconhecidamente os com maiores níveis de atuação. A relação com o endividamento público apresentou sentido contrário ao esperado com base na literatura, mostrando um novo achado neste estudo.

Aárea de educação demonstrou ser a mais afetada pela corrupção nos estados brasileiros. Conforme apresentado por Mauro (1998), esse resultado é preocupante, uma vez que a redução de investimentos em educação reduz o capital humano e, consequentemente, a geração de renda, causando prejuízos ao desenvolvimento socioeconômico do país.

O nível de alfabetização da população é maior nos estados com menores níveis de corrupção, confirmando a expectativa teórica de que o nível de educação condiciona o controle social, o que, por sua vez, tem impacto positivo no controle da corrupção.

O estudo encontrou limitações em mensurar a corrupção, pois ainda são poucas as informações divulgadas com relação às práticas de corrupção. Tal fato é explicado pelo aspecto político, e indica a necessidade de maior transparência na gestão pública com relação a essas informações, com o intuito, principalmente, de viabilizar o controle social. Outros aspectos também são importantes para possibilitar o controle da corrupção, como questões legais, operacionais e de sistemas de informação, que precisam ser melhorados para dar suporte à divulgação de informações.

Outra limitação importante de ser destacada diz respeito à metodologia utilizada, uma vez que as condenações judiciais podem apontar maior interesse em combater as práticas corruptas e não necessariamente sua maior incidência.

Os resultados deste trabalho indicam a necessidade de estudos mais aprofundados, principalmente em estados das regiões Nordeste e Sudeste, buscando identificar o que ocasiona os maiores níveis de corrupção; e, nos estados do Sul e no Acre e em Rondônia, no intuito de descobrir praticas ou condicionantes que possibilitam o controle da corrupção.

De modo geral, pode-se concluir que o nível corrupção é consideravelmente elevado nos estados brasileiros, apesar da tendência de redução apresentada no ano de 2012. Tal fato indica a necessidade de intensificação das medidas governamentais de combate à corrupção para reduzir as distorções de investimentos públicos, principalmente na área social, e proporcionar condições de desenvolvimento socioeconômico do país. 


\section{REFERÊNCIAS}

ABED, M. G. T.; GUPTA, M. S. Governance, corruption, and economic performance. Washington: International Monetary Fund, 2002.

ANDERSON, C. J.; TVERDOVA, Y. V. Corruption, political allegiances, and attitudes toward government in contemporary democracies. American Journal of Political Science, [S. I.], v. 47, n. 1, p. 91-109, 2003. DOI: 10.1111/1540-5907.00007

ARANHA, A. L. M. A percepção de corrupção no Brasil: cidadãos x servidores públicos. Revista Andina de Estudios Políticos, [ S. I.], v. 3, n. 1, 2013.

AVRITZER, L.; FILGUEIRA, F. Corrupção e controles democráticos no Brasil. Brasília: CEPAL/IPEA. 2011. (Texto para Discussão, 32).

BARDHAN, P. Corruption and development: a review of issues. Journal of Economic Literature, [S. I.], v. 35, n.3, p. 1320-1346, 1997.

BOLL, J. L. S. A Corrupção Governamental no Brasil: construção de indicadores e análise da sua incidência relativa nos estados brasileiros. 2010. Dissertação (Mestrado em Economia do Desenvolvimento) - Pontifica Universidade Católica do Rio Grande do Sul, Porto Alegre, 2010.

BROOKS, R. C. The nature of political corruption. Political Science Quarterly, [S. I.], v. 24, n. 1, p. 1-22. 1909.

CARRARO, A.; MACHADO, I. B.; CANEVER, M. D.; BOLL, J. L. S. Proposta para a estimação da corrupção regional no Brasil. Política \& Sociedade, [S. I.], v. 14, n. 31, p. 326-352, 2015. DOI: 10.5007/2175-7984.2015v14n31p326

CROIX, D.; DELAVALLADE, C. Growth, public investment and corruption with failing institutions. Economics of Governance, [S. I.], v. 10, n. 3, p. 187-219, 2009. DOI: 10.1007/s10101-008-0057-4

CUNHA, N. R. D. S.; LIMA, J. E. D.; GOMES, M. F. D. M.; BRAGA, M. J. A intensidade da exploração agropecuária como indicador da degradação ambiental na região dos Cerrados, Brasil. Revista de Economia e Sociologia Rural, [S. I.], v. 46, n. 2, p. 291-323, 2008. DOI: 10.1590/S0103-20032008000200002

DAMATTA, R. Carnavais, malandros e heróis: para uma sociologia do dilema brasileiro. Rio de Janeiro: Rocco. 1997.

DEL MONTE, A.; PAPAGNI, E. Public expenditure, corruption, and economic growth: the case of Italy. European Journal of Political Economy, [S. I.], v. 17, n. 1, p. 1-16, 2001. DOI: 10.1016/S0176-2680(00)00025-2

DEL MONTE, A.; PAPAGNI, E. The determinants of corruption in Italy: Regional panel data analysis. European Journal of Political Economy, [S. I.], v. 23, n. 2, p. 379-396, 2007. DOI: 10.1016/j.ejpoleco.2006.03.004

DELAVALLADE, C. Corruption and distribution of public spending in developing countries. Journal of Economics and Finance, [S. I.], v. 30, n. 2, p. 222-239, 2006. DOI: 10.1007/BF02761488

DEPKEN, C. A.; LAFOUNTAIN, C. L. Fiscal consequences of public corruption: Empirical evidence from state bond ratings. Public Choice, [S. I.], v. 126, n. 1/2, p. 75-85, 2006. DOI: 10.1007/s11127-006-4315-0

FÁVERO, L. P.; BELFIORE, P., Silva, F. D.; CHAN, B. L. Análise de dados: modelagem multivariada para tomada de decisões. Rio de Janeiro: Campus, 2009.

FERES JÚNIOR, João; SASSARA, Luna de Oliveira. Corrupção, escândalos e a cobertura midiática da política. Novos Estudos CEBRAP, [S. I.], v. 35, n. 2, p. 205-225, 2016. DOI: 10.25091/S0101-3300201600020011

FERREIRA, M. A. S. Perspectivas do controle social: uma análise da natureza e extensão da participação cidadã na dinâmica dos municípios mineiros. 2015. 109f. Dissertação (Mestrado em Administração) - Universidade Federal de Viçosa, Viçosa, 2015.

FILGUEIRAS, F. A tolerância à corrupção no Brasil: uma antinomia entre normas morais e prática social. Opinião Pública, [S. I.], v. 15, n. 2, p. 386-421, 2009. DOI: 10.1590/S0104-62762009000200005 
FILGUEIRAS, F.; ARANHA, A. L. M. Controle da corrupção e burocracia da linha de frente: regras, discricionariedade e reformas no Brasil. Dados-Revista de Ciências Sociais, [S. I.], v. 54, n. 2, 2011. DOI: 10.1590/S0011-52582011000200005

FISMAN, R.; MIGUEL, E. Corruption, norms, and legal enforcement: Evidence from diplomatic parking tickets. Journal of Political Economy, [S. I.], v. 115, n. 6, p. 1020-1048, 2007. DOI: 10.1086/527495

HAIR JR, J. F.; BLACK, W. C.; BABIN, B. J.; ANDERSON, R. E.; TATHAM, R. L. Análise multivariada de dados. Porto Alegre: Bookman, 2009.

HOLANDA, S. B. Raízes do Brasil. São Paulo: Companhia das Letras. 1995.

KAUFMANN, D.; KRAAY, A.; MASTRUZZI, M. The worldwide governance indicators: methodology and analytical issues. Hague Journal on the Rule of Law, [S. I.], v. 3, n. 2, p. 220-246, 2011. DOI: 10.1017/S1876404511200046.

LEMOS, J. D. J. S. Níveis de degradação no Nordeste brasileiro. Revista Econômica do Nordeste, [S. I.], v. 32, n. 3, p. 406-429, 2001.

LINDSTEDT, C.; NAURIN, D. Transparency is not enough: Making transparency effective in reducing corruption. International Political Science Review, [S. I.], v. 31, n. 3, p. 301-322, 2010. DOI: 10.1177/0192512110377602

LOPES, L. S.; TOYOSHIMA, S. H. Evidências do impacto da corrupção sobre a eficiência das políticas de saúde e educação nos estados brasileiros. Planejamento e Políticas Públicas, n. 41, p. 199-228, jul./dez. 2013.

MADEIRA, Lígia Mori; GELISKI, Leonardo. O combate a crime de corrupção pela Justiça Federal da Região Sul do Brasil. Revista de Administração Pública, [S. I.], v. 53, n. 6, p. 987-1010, 2019. DOI: 10.1590/0034-761220180237

MAURO, P. Corruption and the composition of government expenditure. Journal of Public Economics, [S. I.], v. 69, n. 2, p. 263-279, 1998. DOI: 10.1016/S0047-2727(98)00025-5

MELO, C. A. V. Corrupção e políticas públicas: uma análise empírica dos municípios do Brasil. 2010. Tese (Doutorado em Ciência Política) - Universidade Federal de Pernambuco, Recife, 2010.

MELO, Felipe Luiz Neves Bezerra de; SAMPAIO, Luciano Menezes Bezerra; OLIVEIRA, Renato Lima de. Corrupção burocrática e empreendedorismo: uma análise empírica dos estados brasileiros. Revista de Administração Contemporânea, [S. I.], v. 19, n. 3, p. 374-397, 2015. DOI: 10.1590/1982-7849rac20151611

MINGOTI, S. A. Análise de dados através de métodos de estatística multivariada: uma abordagem aplicada. Belo Horizonte: Editora UFMG, 2007.

Mo, P. H. Corruption and economic growth. Journal of Comparative Economics, [S. I.], v. 29, n. 1, p. 66-79, 2001. DOI: $10.1006 / j c e c .2000 .1703$

NAS, T. F.; PRICE, A. C.; WEBER, C. T. A policy-oriented theory of corruption. American Political Science Review, [S. I.], v. 80, n. 1, p. 107-119, 1986. DOI: $10.2307 / 1957086$

PASCARELLI FILHO, Mario. A nova administração pública: profissionalização, eficiência e governança. São Paulo: DVS, 2011.

PELLEGRINI, L., G, R. Causes of corruption: a survey of cross-country analyses and extended results. Economics of Governance, [S. I.], v. 9, p. 245-263, 2008. DOI: 10.1007/s10101-007-0033-4

PRAÇA, S. Corrupção e reforma institucional no Brasil, 1988-2008. Opinião Pública, [S. I.], v. 17, n. 1, p. 137-162, 2011. DOI: $10.1590 / S 0104-62762011000100005$

ROSE-ACKERMAN, S. Corruption and government: causes, consequences, and reform. Cambridge: Cambridge University Press, 1999.

SERRA, D. Empirical determinants of corruption: A sensitivity analysis. Public Choice, [S. I.], v. 126, n. 1/2, p. 225256, 2006. DOI: 10.1007/s11127-006-0286-4 
SODRÉ, A. C. A.; ALVES, M. F. C. Relação entre emendas parlamentares e corrupção municipal no Brasil: estudo dos relatórios do programa de fiscalização da Controladoria-Geral da União. RAC-Revista de Administração Contemporânea, [S. I.], v. 14, n. 3, 2010. DOI: 10.1590/S1415-65552010000300003

SVENSSON, J. Eight questions about corruption. Journal of Economic Perspectives, [S. I.], v. 19, n. 3, p. 19-42., 2005. DOI: $10.1257 / 089533005774357860$

TANZI, V. Corruption around the world: causes, consequences, scope, and cures. Staff Papers, [S. I.], v. 45, n. 4, p. 559-594, 1998. DOI: $10.2307 / 3867585$

TREISMAN, D. The causes of corruption: a cross-national study. Journal of Public Economics, [S. I.], v. 76, n. 3, p. 99-457, 2000. DOI: 10.1016/S0047-2727(99)00092-4

WITVLIET, M. I.; KUNST, A. E.; ARAH, O. A.; STRONKS, K. Sick regimes and sick people: a multilevel investigation of the population health consequences of perceived national corruption. Tropical Medicine \& International Health, [S. I.], v. 18, n. 10, p. 1240-1247, 2013. DOI: 10.1111/tmi.12177

ZANI, F. B.; SPINELLI, R. Q. Inovação na gestão pública: eficiência com participação? In: Encontro Nacional dos Programas de Pós-Graduação em Administração, 34., 2010, Rio de Janeiro. Anais [...]. Rio de Janeiro: ENANPAD, 2010. p. 1-17.

\section{Contato:}

Anderson de Oliveira Reis

E-mail: andersonccoufv@gmail.com

Fernanda Maria de Almeida

E-mail: falmeida.ufv@gmail.com 